\title{
A RADIAL UNIQUENESS THEOREM FOR MEROMORPHIC FUNCTIONS
}

\author{
P. J. RIPPON
}

\begin{abstract}
A classical theorem of Lusin and Privalov states that a meromorphic function in the unit disc, which has radial limit sero on a set which is both of second category and metrically dense in some boundary arc, must vanish identically. We prove below a radial uniqueness theorem which includes the Lusin-Privalov theorem as a special case and which also generalises the BarthSchneider-Tse asymptotic analogue of the F. and $M$. Riess radial uniqueness theorem. The part of the proof relating to Baire category is disposed of by using the Collingwood maximality theorem.
\end{abstract}

1. Introduction. We shall be concerned with functions from the unit disc $U=$ $\{|z|<1\}$ to the Riemann sphere $\Sigma$. For such a function $f$ and any $\zeta$ in $T=\partial U$ we write $C_{\rho}(f, \varsigma)$ to denote the radial cluster set of $f$ at $\varsigma$, i.e. the set of $w$ in $\Sigma$ for which there is a sequence $r_{n}$ in $(0,1)$ such that $r_{n} \rightarrow 1$ and $f\left(r_{n} \zeta\right) \rightarrow w$ as $n \rightarrow \infty$.

THEOREM 1. Let $f: U \rightarrow \Sigma$ be meromorphic, $p: U \rightarrow \Sigma$ be continuous and suppose that $f p^{-1}$ has a continuous extension to the whole of $U$. If $S$ and $M$ are second category and metrically dense subsets, respectively, of an arc in $T$ such that

$$
\begin{gathered}
\infty \notin C_{\rho}\left(f p^{-1}, \varsigma\right), \quad \varsigma \in S, \\
\infty \notin C_{\rho}(p, \varsigma), \quad \varsigma \in S,
\end{gathered}
$$

and

$$
0 \in \bigcap_{\varsigma \in M} C_{\rho}(p, \varsigma)
$$

then $f \equiv 0$.

Theorem 1 contains a number of earlier radial uniqueness theorems.

COROLlARY 1. Let $f$ be meromorphic in $U$ and have radial limit zero on a set which is both metrically dense and of second category in an arc of $T$. Then $f \equiv 0$.

This is the well-known Lusin-Privalov radial uniqueness theorem [5]. It follows from Theorem 1 by putting $p=f$ and $S=M$. A generalisation of the LusinPrivalov theorem due to Collingwood and, independently, Bagemihl and Seidel [2, Theorem 8.3] also follows from Theorem 1 in a rather similar way.

Another special case is

COROLlaRY 2. Let $f$ be meromorphic in $U$ and let $p$ be real-valued and monotone decreasing in $[0,1)$ with $p(r) \rightarrow 0$ as $r \rightarrow 1$. If $S$ is of second category in $T$ and

$$
|f(r \varsigma)|=o(p(r)), \quad r \rightarrow 1, \varsigma \in S,
$$

then $f \equiv 0$.

Received by the editors October 29, 1981.

1980 Mathematics Subject Classification. Primary 30D40.

(Q) 1982 American Mathematical Society 0002-9939/81/0000-0267/802.00 
A result of this type was first proved by Barth and Schneider [1] with $f$ bounded and analytic. The extension to meromorphic functions was made by Tse [7].

To deduce Corollary 2 from Theorem 1 put $p(z)=p(|z|), z \in U$, and $M=T$. We may assume that $p$ is continuous. Clearly (1.1), (1.2), and (1.3) are satisfied so that $f \equiv 0$.

If $f$ is taken to be analytic in $U$ then a strengthened version of Corollary 2 can be given which is not a special case of Theorem 1 .

THEOREM 2. Let $f$ be analytic in $U$ and suppose that $p:[0,1) \rightarrow(0, \infty]$ is continuous in the extended sense and satisfies

$$
\int_{0}^{1} \log ^{+} \log ^{+} p(r) d r<\infty
$$

and

$$
\varliminf_{r \rightarrow 1} p(r)=0 .
$$

If $S$ is of second category in $T$ and

$$
|f(r \zeta)|=O(p(r)), \quad r \rightarrow 1, \varsigma \in S,
$$

then $f \equiv 0$.

We remark that results analogous to Theorems 1 and 2 can be proved for subharmonic functions in, for example, a half-space (see [6] where subharmonic analogues of Corollaries 1 and 2 were given).

2. Proofs. In results of the above type the part of the proof relating to category can often be disposed of by appealing to the Collingwood maximality theorem. To state this we introduce the complete cluster set $C(f, \varsigma)$ of a function $f: U \rightarrow \Sigma$ at a point $\zeta$ in $T$, i.e. the set of $w$ in $\Sigma$ for which there is a sequence $z_{n}$ in $U$ such that $z_{n} \rightarrow \zeta$ and $f\left(z_{n}\right) \rightarrow w$ as $n \rightarrow \infty$.

LeMma (Collingwood [2, TheOREM 4.8]). If $f: U \rightarrow \Sigma$ is continuous then

$$
C_{\rho}(f, \varsigma)=C(f, \varsigma)
$$

for all $S$ in $T$ apart from a set of the first category.

To prove Theorem 1 we note that, by the lemma, there exists $S$ in $S$ such that $C_{\rho}\left(f p^{-1}, \varsigma\right)=C\left(f p^{-1}, \varsigma\right)$ and $C_{\rho}(p, \varsigma)=C(p, \varsigma)$. Thus, by (1.1) and (1.2), there is a set $\Omega=U \cap\{|z-s|<\delta\}, \delta>0$, and constants $K, L$ such that

$$
|f(z)| \leq K|p(z)| \leq L, \quad z \in \Omega \text {. }
$$

In particular $f$ is bounded and analytic in $\Omega$. Thus $f$ has finite angular limits almost everywhere on $\Gamma=\partial \Omega \cap T$ which can only be constant on sets of length zero, unless $f$ is identically constant. These assertions follow from Fatou's theorem and the F. and M. Riesz radial uniqueness theorem [2, Theorems 2.4 and 2.5] by using a conformal mapping of $\Omega$ onto $U$. We deduce from (1.3), (2.1) and the fact that $M$ has positive length in $\Gamma$ that $f \equiv 0$ in $\Omega$ and so throughout $U$.

REMARK. It is clear that in Theorem 1 some generalisation of (1.1), (1.2) and (1.3) is possible. Let $\left\{A_{\zeta}\right\}, \varsigma \in T$, denote a family of subsets of $U$ for which the above lemma holds, i.e. for any continuous $f: U \rightarrow \Sigma$, the cluster set of $f$ at $\zeta$ along 
$A_{\zeta}$ equals the complete cluster set of $f$ at $\zeta$ apart from a set of $\zeta$ of first category. Then we can use the cluster sets along the sets $A_{\zeta}$ in (1.1) and (1.2) in place of the radial cluster sets. In this way we obtain a generalisation of the version of Corollary 2 given recently by Gavrilov and Mirzoyan [4, Theorem 4]. The author is grateful to Karl Barth and the referee for bringing this paper to his attention and he hopes to return in a later paper to a more detailed description of the families of sets $\left\{A_{\varsigma}\right\}$, $\varsigma \in T$, for which the lemma holds.

In (1.3), on the other hand, we can replace $C_{\rho}(p, \varsigma)$ by $C_{\mathcal{A}}(p, \varsigma)$, the outer angular cluster set of $p$ at $\varsigma$, i.e. the set of $w$ in $\Sigma$ for which there is a sequence $z_{n}$ in $U$ which approaches $\zeta$ nontangentially so that $p\left(z_{n}\right) \rightarrow w$ as $n \rightarrow \infty$.

To prove Theorem 2 we note that, by the lemma, there exist numbers $K, \delta$ and $\theta_{0}$ such that

$$
\left|f\left(r e^{i \theta_{0}}\right)\right| \leq K p(r), \quad 1-2 \delta<r<1,\left|\theta-\theta_{0}\right|<2 \delta .
$$

By (1.5) there is an increasing sequence $r_{n}, n=1,2, \ldots$, in $(1-\delta, 1)$ such that $r_{n} \rightarrow 1$ as $n \rightarrow \infty$ and

$$
K p\left(r_{n}\right) \leq n^{-1}, \quad n=1,2, \ldots
$$

If

$$
\Omega^{*}=\left\{r e^{i \theta}:|r-1|<2 \delta,\left|\theta-\theta_{0}\right|<2 \delta\right\}
$$

and

$$
u_{n}(z)= \begin{cases}\log ^{+}|f(z)|, & z \in \Omega^{*} \cap\left\{|z|<r_{n}\right\}, \\ 0, & z \in \Omega^{*} \cap\left\{|z| \geq r_{n}\right\},\end{cases}
$$

then the functions $u_{n}$ are subharmonic in $\Omega^{*}$ and satisfy $u_{n}(z) \leq \lambda(|z|), z \in \Omega^{*}$, where

$$
\lambda(r)= \begin{cases}\log ^{+} K p(r), & r<1, \\ 0, & r \geq 1 .\end{cases}
$$

In view of (1.4) we can apply the 'loglog theorem' in its form due to Domar [3, Theorem 3] to see that the functions $u_{n}$ are uniformly bounded above in

$$
\Omega=\left\{r e^{i \theta}: 1-\delta<r<1,\left|\theta-\theta_{0}\right|<\delta\right\} .
$$

Thus $f$ is bounded in $\Omega$ and the proof is easily completed using (2.2) and (2.3).

\section{REFERENCES}

1. K. F. Barth and W. J. Schneider, An asymptotic analogue of the $F$. and $M$. Riesz radial uniqueness theorem, Proc. Amer. Math. Soc. 22 (1969), 53-54.

2. E. F. Collingwood and A. J. Lohwater, Cluster sets, Cambridge Univ. Press, 1966.

3. Y. Domar, On the existence of a largest subharmonic minorant for a given function, Ark. Mat. 3 (1954-58), 429-440.

4. V. I. Gavrilov and M. M. Mirzoyan, Boundary uniqueness theorems for meromorphic functions, Moscow Univ. Math. Bull. 34 (1979), 33-37.

5. N. N. Lusin and I. I. Privalov, Sur l'unicité et la multiplicité des fonctions analytiques, Ann. Sci. Ecole Norm. Sup. (3) 12 (1925), 143-191.

6. P. J. Rippon, The boundary cluster sets of subharmonic functions, J. London Math. Soc. (2) 17 (1978), 469-479.

7. K. F. Tse, An analog of the Lusin-Privalof radial uniqueness theorem, Proc. Amer. Math. Soc. 25 (1970), 310-312.

Department of MAThematics, UNIVERstTy College, CoRK, IRELAND

Department of Mathematics, University of Maryland, College Park, MARYLAND 20742 